\title{
Energy Efficient Cluster Head Selection using Hybrid Squirrel Harmony Search Algorithm in WSN
}

\author{
N. Lavanya ${ }^{1}$, T. Shankar ${ }^{2}$ \\ Department of Communication Engineering, School of Electronics Engineering \\ Vellore Institute of Technology, Vellore, Tamil Nadu, India
}

\begin{abstract}
The Wireless Sensor Network (WSN) has found an extensive variety of applications, which include battlefield surveillance, monitoring of environment and traffic, modern agriculture due to their effectiveness in communication. Clustering is one of the significant mechanisms for enhancing the lifespan of the network in WSN. This clustering scheme is exploited to improve the sensor network's lifespan by decreasing the network's energy consumption and increasing the stability of the network. The existing cluster head selection algorithm suffers from the inconsistent tradeoffs between exploration exploitation and global search constraints. Therefore, in this research, the hybridization of two popular optimization algorithms, namely, Harmony Search Algorithm (HSA) and Squirrel Search Algorithm (SSA) is executed for optimal selection of cluster heads in WSN with respect to distance and energy. The proposed Hybrid Squirrel Harmony Search Algorithm (HSHSA) is found to be energy efficient when compared with first node death (FND) and last node death (LND) of existing Cluster Head Selection (CHS) techniques. In addition to this, the proposed HSHSA shows enhancements in overall throughput and residual energy of the wireless sensor network by $31.02 \%$ and $85.69 \%$, respectively than the existing algorithms.
\end{abstract}

Keywords-Cluster head selection; clustering; harmony search algorithm; squirrel search algorithm; wireless sensor network

\section{INTRODUCTION}

As an improved and innovative data acquisition and processing technology, Wireless Sensor Network (WSN) has an extensive variety of applications in environmental monitoring, military, space exploration, smart furniture, etc. [1]. A WSN can be defined as an autonomous system comprising of a lot of sensor nodes developed for intercommunicating through wireless radio [2]. Energy efficiency is an important aspect of the WSN. Performing routing on a hierarchical basis is a fundamental procedure to decrease the energy consumption in a network [32]. On the other hand, since the cluster heads $(\mathrm{CHs})$ nearer to the base station (BS) are burdened with intense traffic, they drain quicker compared to the rest of the nodes in the cluster. The sensor network algorithms and protocols must be capable of self-organizing. Such a protocol design will improve network lifetime and utilize maximum node energy [10]. In WSN, clustering is a significant routing approach, which can reconfigure the network. WSN is divided into clusters, each possessing a coordinator node referred to as the $\mathrm{CH}$, which is accountable for collecting the information from all the members of the cluster and transmitting the gathered information to the base station called the sink node. Sensors are often deployed densely to satisfy the coverage requirement, which enables specific nodes to go to the sleep mode, thus, saving significant energy [11,31]. The cluster heads are chosen either randomly or by checking for one or more criteria. The technique employed for the selection of cluster heads brings a major impact to the lifetime of the WSN. The node which has the highest count of neighbor nodes, the minimum distance from BS, and the maximum residual energy is termed as the ideal cluster head for a cluster [3]. Cluster Heads can aggregate, process and filter the information forwarded by the member nodes of the cluster. This reduces the network bandwidth and conserves energy consumption. Thus, an efficient selection of cluster heads will improve the network's lifespan and maximizes the energy of the sensor nodes. This cluster based reconfiguration of the network is performed based on the desired requirements.

One of the inflexible demands of the nodes in WSN is the effective use of the energy being stored. Numerous techniques have been developed for managing the energy of nodes in WSN efficiently through different clustering techniques [4]. When the search for an optimum solution of the $\mathrm{CH}$ becomes exhaustive, meta-heuristic optimizations are preferred. To name a few, Particle Swarm Optimization (PSO), Harmony Search Algorithm (HSA), and Squirrel Search Algorithm (SSA) are such meta-heuristic search optimization algorithms employed for finding an optimal solution. The HSA suffers from the drawback of being bound to only a specific search area. Nevertheless, in PSO, the particle traverses from area to area in search of an optimal solution. However, the PSO suffers from the issue with exploitation and exploration in the problems involving high dimensionality and in the problems which consume a long computational time for achieving local minima or maxima [5].

\section{A. Our Contribution}

In contrary to the existing algorithms of cluster head selection, this research work integrates the SSA and HSA optimization techniques. The HSA exhibits high searching efficiency. In SSA, the squirrels move from one locality to another locality in search of a globally optimal solution, hence the exploration is not region specific like HSA. In addition to this, the SSA exhibits high convergence property, unlike PSO. Therefore, the proposed HSHSA scheme utilizes high search efficiency of HSA integrated with the high convergence and dynamic property of SSA for selecting energy efficient cluster heads for the WSN. The important objectives of this research are: 
- To deploy nodes in the WSN and form clusters.

- To develop HSHSA optimization for Cluster Head Selection (CHS).

- To improve the overall throughput and residual energy of nodes in the modeled WSN.

- To validate the improved performance of the proposed HSHSA CHS mechanism.

The remaining sections of the research paper are organized in the following manner: Section 2 includes the discussion of the works done by the researchers previously; Section 3 explains the methodology proposed; Section 4 presents all the simulation results and their inferences, and Section 5 provides a conclusion as well as a scope to extend the work in the future.

\section{LITERATURE REVIEW}

The literature review section of the paper includes the works proposed previously for selecting the cluster heads namely, LEACH protocol, NSGA, PSO, GSA, ABC, HSA, etc. In [7], the authors analyze a scheme that integrates four important clustering techniques such as connectivity based scheme ID based clustering, weighted approach, and probability based clustering. Ant Colony Optimization (ACO) is employed for optimizing the count of clusters and for selecting the optimal cluster heads. However, the time of convergence is very uncertain for ACO algorithms.

The authors in [12] have provided an HSA scheme which is based on the centralized cluster-dependent mechanism. Here, this approach is dependent on music and is a Meta-heuristic optimization technique, which decreases the distance. This method subsists among the cluster heads in addition to the associated nodes, leading to the optimization of energy distribution in the sensor network. However, the HSA method of optimization, the search for an optimal solution is stuck in the local minima. The authors in [13] proposed a modified synchronous FireFly algorithm for the selection of cluster heads in WSN. The Firefly algorithm is a heuristic search optimization technique. This was done to resolve the uneven network degradation and to improve network performance. The results are better compared to the performance of LEACH and conventional firefly technique; however, the lifetime for the nodes can still be improved.

The authors in [14] have examined a PSO clustering method that could enhance the lifetime of the network by disposing of the formation of single nodes. Hence, they suggested an E-OEERP that worked based on both the GSA and PSO. Here, the PSO was employed for eliminating the formation of nodes and the GSA scheme was used for the selection of routes. However, the cluster overhead was the main disadvantage of the clustering approach. The authors in [15] worked on a clustering scheme to improve the network lifetime. Here, a modification to the LEACH algorithm was proposed which worked based on the shortcomings of original LEACH by exploiting the distance measured with the current node was proposed. Hence novel thresholds from node energy as well as the distance factors of the individual node have been developed. But this protocol does not solve the random distribution issue of $\mathrm{CHs}$ as well as cluster dimensions [8]. The authors in [16] presented a review on scheduling mechanisms that focused on improving the energy efficiency in WSN having a different architecture of the network compared to the conventional WSN. In order to decrease the whole time taken for transmission and extend the lifespan of the network, the conventional sensor network is substituted by SDN architecture.

The authors in [18] proposed a PSO algorithm for implementing CHS in WSN in an energy efficient manner. This method comprises of efficient fitness function and particle encoding technique. Residual energy, sink distance, and intracluster distance are the parameters considered for optimization. The drawback of the PSO technique is the problem of exploration and exploitation. The authors in [19] have presented an $\mathrm{ABC}$ meta-heuristic with an enhanced solution search equation to enhance its utilization capability. An enhanced population sampling approach was developed. It needs a single controlling parameter to calculate and save the result. Thus, it increases the efficacy of the suggested methodology. A fine balance between exploitation and exploration was maintained by the use of a proposed approach with minimum memory requirements. However, the $A B C$ algorithm is slow while processing sequential data. The authors in [30] proposed a cluster head selection scheme to improve the network lifetime using hybridization of firefly and grey wolf optimization algorithm. The main motive of this research is to decrease the overhead and stabilize the network. However, the lifetime for the nodes can still be improved.

The authors in [6] suggested the sorting of results of multiobjective problems in a non-dominated manner by the use of a multi-objective evolutionary algorithm (MOEA), referred by Non-dominated Sorting Genetic Algorithm II (NSGA-II) for prolonging the lifespan of the WSN. The optimization of the consumption of energy is carried out for enhancing the lifetime of the network. The drawback of this approach is that the nodes in the network start dying from the initial rounds. The authors in [9] presented an energy efficient scheme of hierarchical clustering for WSN. The nodes form clusters in a hierarchical manner for maximizing coverage and minimizing energy consumption. This method of clustering is decentralized. As an extension, this approach can be formulated as a centralized clustering scheme for improving energy efficiency.

The authors in [17] suggested a spectrum sensing mechanism based on clusters, for minimizing energy consumption. The formation of clusters is implemented by the Fuzzy C-means approach and the $\mathrm{CHs}$ are chosen based on locations of sensors which are the members of clusters. However, this method causes nodes to die constantly throughout. The authors in [20] proposed an algorithm for routing which integrates cluster formation, CHS, and multipath routing for data transmission. As a result, energy consumption and routing overheads are decreased. The best path is selected using a Genetic Algorithm, which considers minimal energy dissipation and distance in the objective function. This increases the lifetime of the network by sharing the traffic evenly for all the nodes. From the study of the literature, this research proposes the hybridization of HSA and SSA optimization technique for the energy efficient and optimal selection of cluster heads. The proposed HSHSA technique 
resolves the problem found in the previously proposed algorithms such as exploration and exploitation and improves the search efficiency and convergence rate.

\section{RESEARCH METHODOLOGY}

\section{A. Network Model}

The free space network model is taken into consideration for this study. It comprises a receiver section and a transmitter module with $\mathrm{d}$ as the separation distance. The transmitter module contains transmission electronics with an amplifier for the transmission and the receiver module contains receiving electronics as a portion for data to be communicated using bits. The network model is assumed to be a group of sensor nodes scattered on a field that is in the shape of a rectangle [21]. The communication energy of the sensor network is calculated using the following equation (1-2). The below mentioned properties are considered for modeling the WSN:

- The sensor nodes in the WSN are assumed to be in the quasi-stationary state.

- Nodes are not aware of their location.

- The sensor nodes in the modeled WSN are in a homogeneous fashion.

- The consumption of energy for the sensor nodes in the network is non-uniform in nature and is dependent on the distance of the sensor node from the cluster head or BS.

- There is no need for monitoring of the nodes after deployment since these nodes are self-organizing. The power levels of all the nodes are fixed.

$E_{T x}(l, d)=\left\{\begin{array}{c}l E_{\text {elec }}+l E_{f s} d^{2}, \quad d<d_{0} \\ l E_{\text {elec }}+A l E_{m p} d^{4}, \quad d \geq d_{0}\end{array}\right.$

$\mathrm{E}_{\mathrm{Rx}}(\mathrm{l})=1 \mathrm{E}_{\text {elec }}$

where $E_{R x}$ denotes the energy consumed by the receiver for the message of bit length 1 , and $E_{T x}$ indicaes the energy consumed by the transmitter for that same message. $E_{\text {elec }}$ is the electronics energy, which is dependent on the parameters namely, modulation, spreading of the signal, filtering, and digital coding, while the amplifier energy $E_{f s} d^{2}$ (free space model) or $E_{m p} d^{4}$ (multi-path model) relies on the distance from the node to the receiver, and the permissible level of biterror rate. The separation distance is defined using the following equation (3).

$\mathrm{d}=\cos \theta=\frac{\mathrm{A} \cdot \mathrm{B}}{\|\mathrm{A}\|\|\mathrm{B}\|}$

where $\mathrm{A}$ and $\mathrm{B}$ are the co-ordinates of the cluster head $\mathrm{CH}$ and the node $\mathrm{i}$, respectively. In the considered WSN, n number of sensors are randomly arranged in a field of area ' $\mathrm{M} \times \mathrm{N}^{\prime} \mathrm{m}^{2}$. For optimizing the approach of selecting $\mathrm{CHs}$ and for determining the optimal ' $\mathrm{k}$ ' $\mathrm{CHs}$, the objective function employed for computing the optimal solution is given in equation (4) [12]: $\mathrm{f}_{\text {obj }}=\epsilon \times \mathrm{f}_{1}+(1-\epsilon) \times \mathrm{f}_{2}$

where $f_{1}$ and $f_{2}$ are given by

$f_{1}=\max _{k}\left\{\sum_{\forall,{\text { node }, i \in C_{k}}_{k}} \frac{d\left(\text { node }_{i}, C_{k}\right)}{\| \text { luster }_{k} \|}\right\}$

$f_{2}=\frac{\sum_{i=1}^{N} E\left(\text { node }_{i}\right)}{\sum_{j=1}^{k} E\left(\mathrm{CH}_{j}\right)}$

where the scaling factor $(\epsilon)$ ranges between 0 to 1 . In the fitness function given in (4), $f_{l}$ is the largest separation distance of the cluster members 'node $\forall_{i} \in$ node cluster Cluster $_{k}$ ' to their corresponding cluster head $\mathrm{CH}_{k}$ and $\|$ Cluster $_{k} \|$ is the total count of nodes which are the cluster members of Cluster Whereas, $f_{2}$ denotes the ratio of initial energy of all the sensor nodes that are alive, $E\left(\right.$ node $\left._{i}\right)$ in the WSN, to the aggregate of the cluster head's current state energy ' $E\left(C_{k}\right)$ ' of the current iteration of round; $\epsilon$ is a constant that denotes the impact of ' $f_{1}$ ' and ' $f_{2}$ ' in the considered fitness function ' $f_{o b j}$ '.

\section{B. Proposed Methodology}

Harmony Search Algorithm: The Harmony Search Algorithm idealizes the improvisation procedure by a skillful musician. There are three options preferred when a musician improvises: (1) play a similar or known music notes (by altering the pitch to some extent); (2) compose random or new notes; (3) play a popular form of music (a sequence of pitches in the harmony) from the notes of his/her memory. Zong Woo Geem et al. officially announced the above mentioned three choices into a mathematical algorithm of optimization in 2001, and these corresponding elements become pitch adjusting, randomization, and utilization of harmony memory [22]. The utilization of harmony memory is essential, as it is the same as the choice of the best-fit entities in bio-inspired optimizers. This ensures the exploitation of the optimal harmonies in the updated harmony memory. In order to utilize this memory more efficiently, it is generally allotted as a factor $r_{\text {accept }} \in[0,1]$, referred to as the harmony memory considering rate (HMCR). When HMCR is very less, only a small number of best harmonies are chosen and that may lead to very slow convergence. When HMCR is exceptionally high (close to 1), almost all the harmonies are employed in the harmony memory, while the rest of the harmonies are not exploited properly, which results in potentially faulty solutions. Hence, $r_{\text {accept }}$ is defined between 0.7 and 0.95 .

The pitch adjustment is the second element, found using a pitch adjusting rate $r_{p a}$ and a pitch bandwidth $b_{\text {range. Although in }}$ music, adjusting the pitch contributes a lot in changing the frequencies, it relates to producing a slightly varied solution in the HSA. The randomization is the third element, which is to improve the solutions of diversity. Though pitch adjustments play a similar part, it is restrained to several local adjustments of pitches and looks for an optimal solution locally. The usage of random assignment can make the system to additionally exploit several diversified solutions, such that the global optimality is found.

1) Squirrel Search Algorithm: The process of search is initialized when the flying squirrels (FSs) begin scavenging. In warm weather conditions (like autumn), these squirrels scavenge for food sources by flying from one tree to another. 
This activity changes their locations and begins exploration for food in different regions of the forest. Since the weather conditions are adequately hot, the squirrels can fulfill their day to day requirements of food rapidly on the diet of acorn nuts present abundantly and thus the squirrels eat those acorns right after they're found. When the daily necessity is fulfilled, the squirrels will start to search for the optimal source of food in order to store the requirements during winter (hickory nuts). Storing these nuts aids the squirrels to maintain the requirements of energy during intense weather conditions. This will also decrease the expensive foraging, and thereby raise the survival probability. In winter, the loss of leaves in the forest can result in an elevated risk of predator presence and so the squirrels become less active but do not hibernate in that period of the season. When the winter season comes to an end, these squirrels become active again. These actions of scavenging happen recurrently and continue throughout the lifetime of a flying squirrel. This is also the main reason to form the foundation of SSA [29]. The following are the assumptions taken into consideration for the simplification of the mathematical model:

- In a deciduous forest, $\mathrm{n}$ numbers of flying squirrels are considered, out of which only a single squirrel is considered to be on a single tree.

- The entire population of squirrels independently looks for a food source and utilizes the food sources available optimally by demonstrating a dynamic behavior of scavenging.

- In the deciduous forest, three kinds of trees exist; they are normal trees, hickory nut trees, and oak trees (acorn nut trees).

- The area of the forest under the assumption is considered to comprise one hickory tree and three oak trees.

2) Proposed HSHSA for Optimal Cluster Head Selection: The proposed approach of CHS, called the HSHSA, relies on the general attributes of HSA as well as SSA optimization schemes. The optimization technique HSA is capable of producing solutions and metrics of PAR and HMCR, to permit the solution to evade from the optimum solution obtained locally and in order to enhance the optimum solution obtained globally. Because of this reason, HSA is integrated with the SSA to resolve the problem of exploitation and exploration in high dimensionality, thereby achieve optimal solution with good rate of convergence accuracy. The flow diagram of the proposed HSHSA scheme optimal CHS is displayed in the Fig. 1.

In HSHSA optimization, employing SSA permits the squirrels to navigate from one area to other area by changing the locations and seasons at the termination of every iteration of round of data transmission. The dynamic behaviour of SSA agrees to look for optimal solution in $\mathrm{n}$ number of regions of the space with higher convergence rate along with high search efficiency of HSA in each area. This functional working of the
HSHSA optimization is executed for several rounds of transmission of data. For every round, the $\mathrm{CHs}$ conduct transmission of data by collecting information from all the members of the cluster and by transferring to the sink.

The following steps are implemented in the HSHSA:

Step 1: Creation of Network Model: Initial WSN is modelled with nodes. And the energy of all the nodes are initialized.

Step 2: Initialization of Parameters: In a forest, it is considered that there are $\mathrm{n}$ number of FS and the position of the $\mathrm{i}^{\text {th }} \mathrm{FS}$ is defined by a matrix. The locations of all the flying squirrels are exemplified in the matrix given below (7):

$F S=\left[\begin{array}{lllll}F S_{1,1} & F S_{1,2} & \ldots & \ldots & F S_{1, d} \\ F S_{2,1} & F S_{2,2} & \ldots & \ldots & F S_{2, d} \\ \vdots & \vdots & \vdots & \vdots & \vdots \\ \vdots & \vdots & \vdots & \vdots & \vdots \\ F S_{n, 1} & F S_{n, 2} & \ldots & \ldots & F S_{n, d}\end{array}\right]$

where $F S_{i, j}$ represents the $\mathrm{i}^{\text {th }} \mathrm{FS}$ in $\mathrm{j}^{\text {th }}$ dimension. An even distribution is required for allocating the initial position of all the flying squirrels present in the forest as given in the following equation (8) [23].

$F S_{i}=F S_{L B}+U(0,1) \times\left(F S_{U B}-F S_{L B}\right)$

where $\mathrm{FS}_{\mathrm{UB}}, \mathrm{FS}_{\mathrm{LB}}$ are upper and lower limits respectively of $i^{\text {th }} \mathrm{FS}$ in the $j^{\text {th }}$ dimension; whereas $\mathrm{U}(0,1)$ refers to the uniformly distributed randomly generated number defined in the interval $[0,1]$. The initialization of flying squirrels and harmony memory in HSHSA optimization is called as Squirrel Harmonic Memory (SHM). The initial SHM comprises of numerous randomly produced results of the optimization problem considered for the study. The result of the problem being formulated is an index of the ' $\mathrm{k}$ ' number of CHs. SHM is calculated using the equation (9). The rows of the SHM matrix denote random solutions for the problem of optimization considered. The fitness value for each Hybrid vector is calculated with the help of equation (4).

$\left[\begin{array}{llll}F s_{1}^{1} & F s_{2}^{1} & \ldots & F s_{k}^{1} \\ F s_{1}^{2} & F s_{2}^{2} & \ldots & F s_{k}^{2} \\ \vdots & \vdots & \ddots & \vdots \\ F S_{1}^{S H M} & F S_{2}^{S H M} & \ldots & F s_{k}^{S H M}\end{array}\right]\left[\begin{array}{l}f_{o b j_{-} 1} \\ f_{o b j_{-} 2} \\ \vdots \\ f_{o b j_{-} n}\end{array}\right]=\left[\begin{array}{l}F^{1} \\ F^{2} \\ \vdots \\ F^{S H M}\end{array}\right]$

Step 3: Sorting, Declaration, and Randomized Selection: Upon obtaining the position fitness values of all the FSs, subsequently they are sorted in an ascending manner. The FS having the minimum value of fitness is assumed to stay on the hickory nut trees. The five successive best FSs are assumed to stay on the acorn nut tree. These five squirrels are supposed to fly toward the direction of hickory nut tree. The rest of the FSs are considered to stay on the normal trees. Furthermore, by performing randomized selection, few FSs are supposed to fly toward the direction of hickory nut tree considering that the squirrels have contented with their regular day to day energy demands. The remaining FSs will glide towards the acorn nut tree. The scavenging activity of these squirrels is constantly 
impacted by the existence of predators. The natural behavior of FSs is developed by formulating the mechanism of updating locations with $\mathrm{P}_{\mathrm{dp}}$, known as the predator presence probability. There are three possible scenarios that can take place in the food scavenging activity of flying squirrels. In each scenario, the following conditions are assumed:
- In the absence of a predator, a FS glides and effectively explores for its favorite food throughout the forest.

- In the presence of a predator, the FS is careful. FSs are enforced to perform a small randomized walk to explore a neighboring location for hiding.

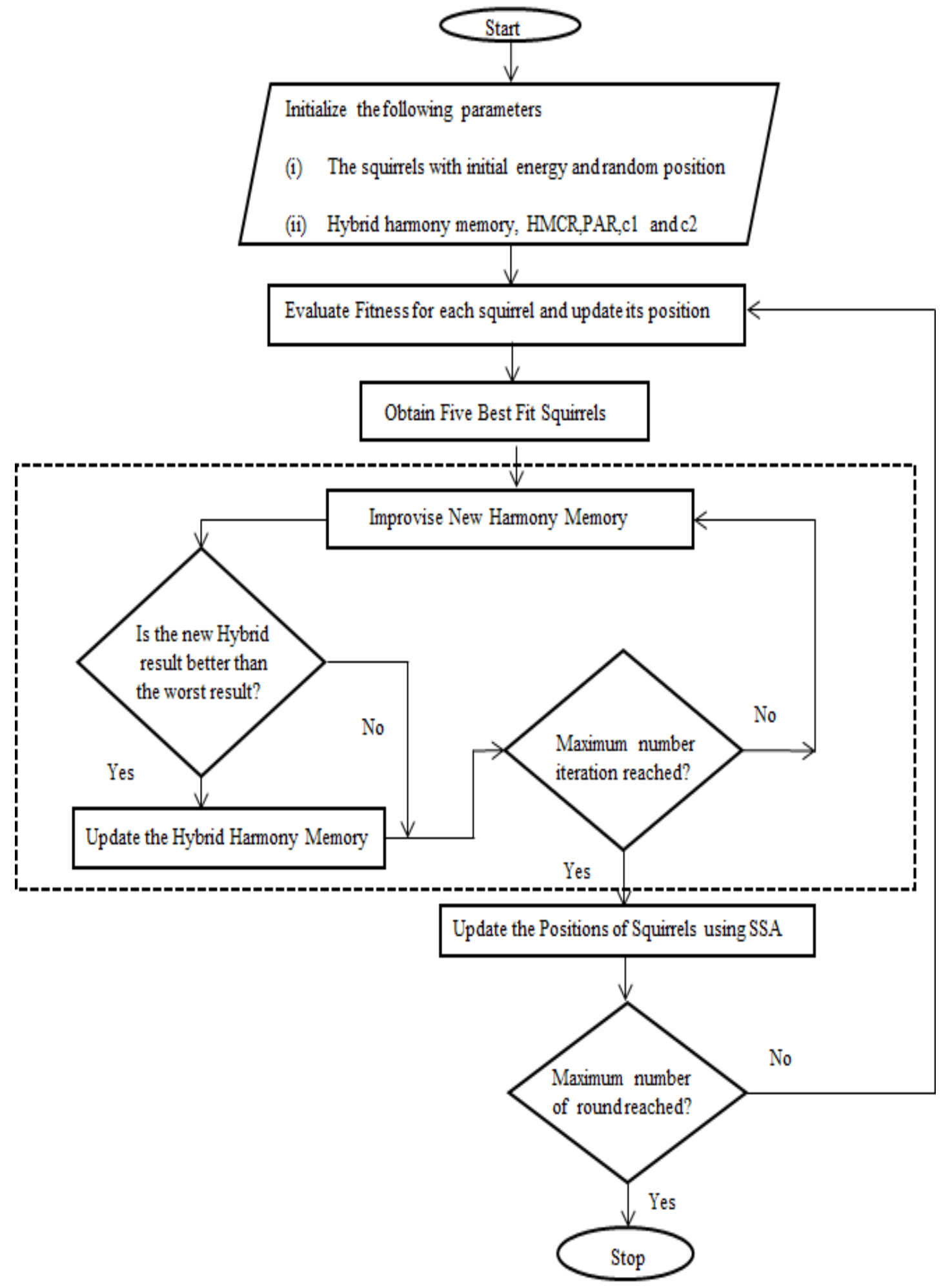

Fig. 1. Flowchart of the Proposed HSHSA. 
The dynamic scavenging activity of the FSs can be modeled mathematically for the three situations in the following manner [23]:

Scenario 1: FSs that are on acorn nut tree (FSat) might glide in the direction of hickory nut trees. On considering this situation, the updated positions of the FSs are attained by equation (10):

$F S_{a t}^{t+1}=\left\{\begin{array}{lr}F S_{a t}^{t}+d_{g} \times G_{c} \times\left(F S_{h t}^{t}-F S_{a t}^{t}\right), & R_{1} \geq P_{d p} \\ \text { Randomlocation, } & \text { otherwise }\end{array}\right.$

where $d_{g}$ is random gliding distance, $R_{1}$ denotes the random number in the range of $[0,1]$, $t$ indicates the current iteration and $F S_{h t}^{t}$ is the position of the flying squirrel which got hold of a hickory nut tree. The balance between exploitation and exploration is attained using the gliding constant $G_{c}$ mentioned in the mathematical model.

Scenario 2: $F S_{n t}^{t}$ denotes the FSs that are on the normal trees, and might fly in the direction of acorn nut tree to meet the regular food demands. On considering this situation, the updated position of FS can be achieved in the following equation (11) [23]:

$F S_{n t}^{t+1}= \begin{cases}F S_{n t}^{t}+d_{g} \times G_{c} \times\left(F S_{a t}^{t}-F S_{n t}^{t}\right), & R_{2} \geq P_{d p} \\ \text { Randomlocation, } & \text { otherwise }\end{cases}$ $[0,1]$.

where $R_{2}$ is a number selected randomly within the interval

Scenario 3: Few FSs from the normal trees that have already consumed acorn nuts might glide in the direction of the hickory nut tree for the purpose of storing the hickory nuts for consuming them when there is scarcity in food. In this situation, the updated positions of FSs can be achieved using the following equation (12) [23]:

$F S_{n t}^{t+1}= \begin{cases}F S_{n t}^{t}+d_{g} \times G_{c} \times\left(F S_{h t}^{t}-F S_{n t}^{t}\right), & R_{3} \geq P_{d p} \\ \text { Randomlocation, } & \text { otherwise }\end{cases}$

where $R_{3}$ denotes a number generated randomly from the interval $[0,1]$. Predator presence probability $P_{d p}$ is assumed as 0.1 .

Step 4: Updating New Harmonic Memory: After declaring the SHM according to the equation (9), the SHM is improved by producing a new vector of harmonies $\left[\mathrm{Fs}_{1}^{\prime}, \mathrm{Fs}_{2}^{\prime}, \ldots, \mathrm{Fs}_{\mathrm{k}}^{\prime}\right]$. Every element of the new harmony matrix denoted by ' $\mathrm{I}_{\mathrm{j}}$ ', is produced with the help of the equation (13) on the basis of the defined HMCR value.

$F s_{j}^{\prime} \leftarrow\left\{\begin{array}{c}F s^{\prime}{ }_{j} \in S H M \text { with probablity } H M C R \\ F s_{j}^{\prime} \in F s_{j} \text { with probability }(1-H M C R)\end{array}\right.$

HMCR is referred to the probability of choosing an element from the SHM elements, and (1-HMCR) is, thus, the possibility of randomly producing it. If $F S_{j}^{\prime}$ is produced by the SHM, then $F s_{j}$ is furthermore altered or otherwise mutated with respect to the PAR value. This finds the possibility of the SHM candidate to be altered and (1-PAR) gives the possibility of an alternative process. The alteration of the pitch for the chosen $F s_{j}{ }_{j}$ can be given as,
$F s_{j}^{\prime} \leftarrow\left\{\begin{array}{l}F s_{j}^{n} \in S H M \text { with probablity } P A R \\ F s_{j}^{\prime} \text { with probability }(1-P A R)\end{array}\right.$

where $F s_{j}^{n}$ refers to a node that is in the closest proximity, having an energy that is higher than the present CHs energy of the respective cluster.

Step 5: Updating the Hybrid Harmony Matrix: This hybrid harmony matrix is generated newly and is assessed with respect to the fitness value obtained for the optimal solution from every row of SSA. If the updated hybrid harmony vector's fitness value is better compared to the fitness value obtained for the SHM's worst harmony, then $\mathrm{n}$ updated harmony is comprised in the SHM as well as the prevailing worst harmony is eliminated from the SHM.

Step 6: Update Locations of Flying Squirrels: Gliding behavior of flying squirrels is defined in terms of gliding equilibrium of which the total sum of drag (D) and lift (L) force generates a resultant force $(R)$ having equal magnitude and a direction opposing the weight $(\mathrm{Mg})$ of the FS. Hence, $\mathrm{R}$ offers a linear route of gliding for the flying squirrel, which is gliding at a persistent velocity (V) [24]. In this research, an approximated prototype of gliding behavior is employed in developing the technique of optimization. A squirrel flying at constant speed permanently descending with an angle $\phi$ to the vertical and lift-to-drag or glide ratio is expressed in the following equation (15) [25].

$\frac{L}{D}=\frac{1}{\tan \phi}$

The FSs can upsurge their flight-path distance by producing minimal glide angle $(\phi)$, thereby increasing the glide ratio. In this work, the lift leads to a downward air deflection above the squirrels' wings. This can be expressed in equation (16) [23]:

$L=\frac{1}{2 \rho C_{L} C^{2} A}$

where $\rho\left(1.204 \mathrm{kgm}^{-3}\right)$ denotes the density of air, $C_{L}$ refers to the coefficient of lift, $\mathrm{C}\left(5.25 \mathrm{~ms}^{-1}\right)$ indicates the speed, whereas A $\left(154 \mathrm{~cm}^{2}\right)$ indicates the surface area of squirrel's body. The foraging of FSs is impacted due to the modifications in the weather and including such activity might offer a realistic methodology towards achieving the optimal solution. Thus, a criterion for monitoring the seasons is established in SSA, which stands as a reason to prevent the proposed technique from being bound to the solutions of local optima. The seasonal constant $\left(\mathrm{S}_{\mathrm{c}}\right)$ is calculated using the following equation (17) [23].

$S_{c}^{t}=\sqrt{\sum_{k=1}^{d}\left(F S_{a t, k}^{t}-F S_{h t, k}\right)^{2}}$

where $t=1,2,3$. Then the search is processed until the criterion $S_{c}^{t}<S_{\min }$ is met. where $S_{\min }$ is the minimal value of seasonal constant calculated using equation (18):

$S_{\text {min }}=\frac{10 E^{-6}}{365^{t /(t m / 2.5)}}$

where $t_{m}$ and $t$ indicate the maximum and current values of iteration, respectively. 
If the criterion for seasonal monitoring is satisfied, then the FSs are randomly relocated which fails to search the forest for an optimum food source for the winter. It is assumed that the squirrels that failed to search the hickory nut tree and still continued to live, will fly to various directions in search of a source of food. The relocation of those FSs is modeled using equation (19) [23]:

$F S_{n t}^{n e w}=F S_{L B}+\operatorname{Lévy}(n) \times\left(F S_{U B}-F S_{L B}\right)$

where Lévy distribution ensures the efficient and better exploration of the considered search area.

Step 7: Stopping Criterion: The procedure from step 4 to 6 is repeated until the maximum number of rounds is reached; otherwise, the algorithm continues to search for optimal solutions. The pseudocode of the proposed HSHSA CHS technique is given as follows.

Pseudocode of Hybrid Squirrel Harmony Search Optimization Algorithm:

Initialize the WSN model with nodes

Initialize the parameter of squirrel search algorithm

$r_{\text {max }} \leftarrow$ Number of iterations for cluster head selection

$N I \leftarrow$ Number of iterations for squirrel search

$N_{S q} \leftarrow$ squirrel search matrix size; $S H M=N_{S q}$

for $t \leftarrow 1$ to $r_{\max }$ do

To construct a random election of normal nodes

for $i \leftarrow 1$ to $N I$ do

for $j \leftarrow 1$ to $N_{S q}$ do

$F S(j,:) \leftarrow$ Randomly chossen cluster head for Squirrel search.

$f_{O b j}(j) \leftarrow$ Fitness values for $F S(j,:)$. By using equation (4)

End

Sorting the rows of $F S$ corresponding to $f_{O b j}$

Declare $F S_{h t}$ and $F S_{a t}$ from the fitness values.

Randomly select three sets by Squirrel search algorithm

Using equations (10) - (12) to update the $F S$

End

Using Harmony search techniques to update the matrix $F S$

Selecting the best Cluster heads from FS for Data Transmission End

\section{RESULTS AND DISCUSSION}

The simulation of the suggested HSHSA approach of CHS is implemented in MATLAB R2018a environment. The parameters of the simulation taken into consideration for the proposed method are given in Table I.

The proposed HSHSA is compared with the existing CHS techniques namely, Direct Transmission, LEACH [26], PSO [27], HSA [28], and SSA [29].

The throughput obtained for different algorithms are shown in the Fig. 2. It can be seen that the throughput of DT drops to zero around 315 rounds. This is because the DT allows all the nodes to communicate directly with the BS. The LEACH protocol shows improvement compared to the DT, where the throughput drops to zero at around 715 rounds because of the randomized selection of cluster heads. Then meta-heuristic search algorithms such as PSO, HSA, and SSA algorithms produce throughput that drops to zero at around 1230, 905, and 1637 rounds respectively. And the proposed HSHSA CHS, combining the advantages of both HSA and SSA produces a throughput that lasts up to 1752 rounds. The proposed HSHSA shows $42.86 \%$ and $21.43 \%$ enhancement in the throughput over the HSA and SSA meta-heuristic algorithms, respectively.

TABLE. I. SIMULATION PARAMETERS

\begin{tabular}{|l|l|}
\hline Parameter & Value \\
\hline Sensor field region $\left(\mathrm{m}^{2}\right)$ & $\left(10 *^{*} 100\right)$ \\
\hline Data packet length $(l)($ bits $)$ & 4096 \\
\hline Initial energy for all the nodes $\left(\mathrm{E}_{\mathrm{o}}\right)(\mathrm{J})$ & 0.5 \\
\hline Number of nodes $(\mathrm{n})$ & 100 \\
\hline $\mathrm{E}_{\text {amp }}\left(\mathrm{pJ} /\right.$ bit/m $\left.{ }^{2}\right)$ & 120 \\
\hline $\mathrm{E}_{\text {elec }}(\mathrm{nJ} / \mathrm{bit})$ & 70 \\
\hline Energy data aggregation $(\mathrm{nJ})$ & 5 \\
\hline Number of search iterations & 5 \\
\hline Number of rounds $\left(\mathrm{r}_{\text {max }}\right)$ & 2000 \\
\hline Number of cluster heads selected $(\mathrm{k})$ & 5 \\
\hline Predator presence probability $\left(\mathrm{P}_{\mathrm{dp}}\right)$ & 0.1 \\
\hline Density of air $(\rho)\left(\mathrm{kg} / \mathrm{m}^{3}\right)$ & 1.204 \\
\hline Speed $(\mathrm{C})(\mathrm{m} / \mathrm{s})$ & 5.25 \\
\hline Surface area of body $(\mathrm{A})\left(\mathrm{cm}{ }^{2}\right)$ & 154 \\
\hline Lift Coefficient $\left(\mathrm{C}_{\mathrm{L}}\right)$ & $0.675 \leq \mathrm{C}_{\mathrm{L}} \leq 1.5$ \\
\hline Drag Coefficient $\left(\mathrm{C}_{\mathrm{D}}\right)$ & 0.6 \\
\hline HMCR & 0.95 \\
\hline
\end{tabular}

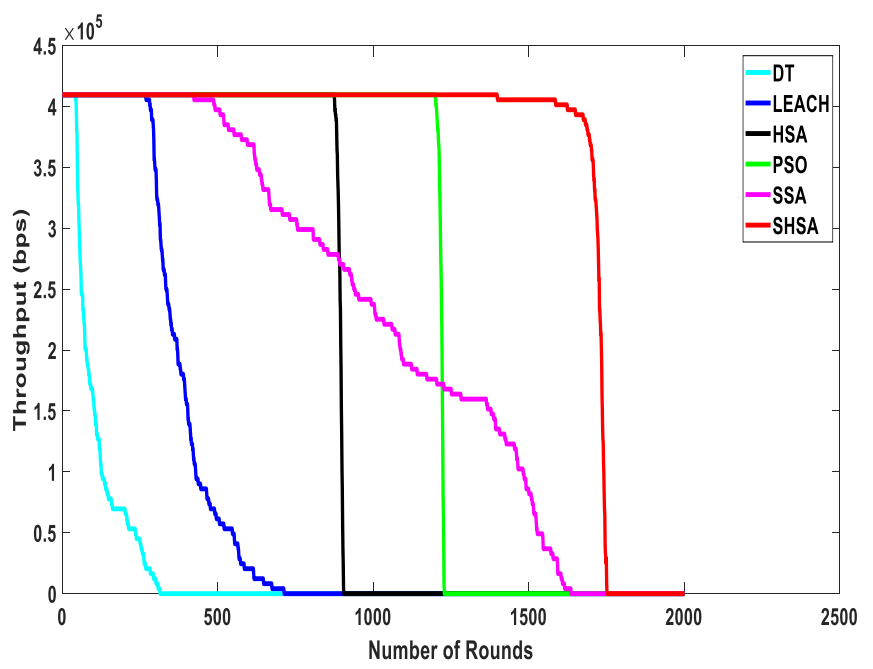

Fig. 2. Comparison of Throughput Obtained for Various Algorithms. 


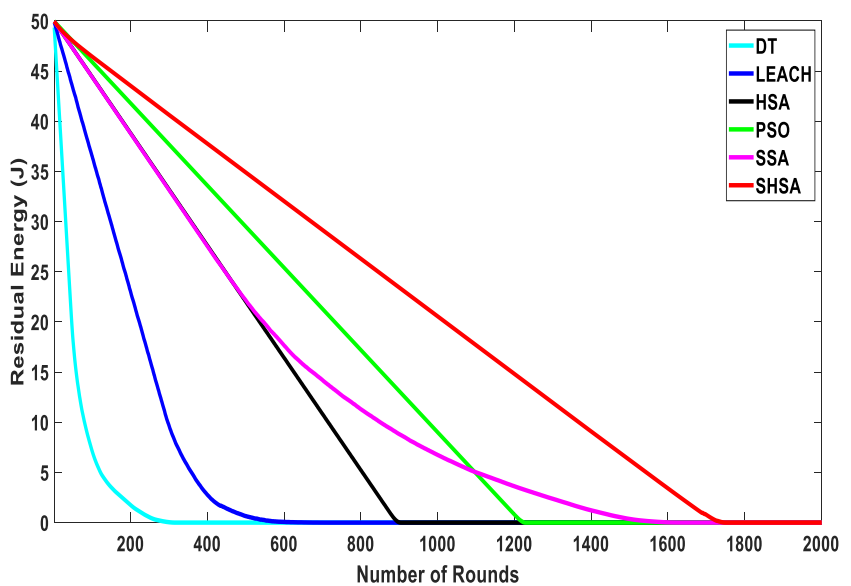

Fig. 3. Comparison of Residual Energy Obtained for Various Algorithms.

The residual energy obtained for different algorithms are shown in the Fig. 3. It can be seen that the residual energy after DT drops to zero at around 315 rounds. This is because the DT allows all the nodes to communicate directly with the BS that causes the nodes to lose energy very quickly. The LEACH protocol shows improvement compared to the DT, where the residual energy drops to zero at around 715 rounds because of the randomized selection of cluster heads. Then meta-heuristic search algorithms such as PSO, HSA, and SSA algorithms show residual energy that drops to zero at around 1230, 905, and 1637 rounds respectively. The proposed HSHSA technique, combining the advantages of both HSA and SSA shows residual energy that lasts up to 1752 rounds. The proposed HSHSA shows $69.84 \%$ and $32.43 \%$ enhancement in the residual energy over the HSA and SSA algorithms, respectively.

The alive nodes sustained for different algorithms are shown in the Fig. 4. It is evident that the nodes stay alive up to 315 rounds for DT. This is because the DT allows all the nodes to communicate directly with the BS that causes the nodes to lose energy very quickly, thereby, causing the nodes to die. The LEACH protocol shows improvement compared to the DT, where the nodes stay alive till 715 rounds because of the random selection of cluster heads. Then meta-heuristic search algorithms such as PSO, HSA, and SSA algorithms maintain the nodes alive up to 1230,905 , and 1637 rounds respectively. And the proposed HSHSA technique of CHS, combining the advantages of both HSA and SSA maintains the nodes to stay alive up to 1752 rounds. The proposed HSHSA technique of CHS shows $48.29 \%, 6.57 \%$, and $29.86 \%$ enhancement in maintaining the nodes alive compared to the HSA, SSA, and PSO algorithms, respectively.

Fig. 5 provides the dead nodes for different algorithms. It can be seen that the first node dies at the round 44 and eventually all the nodes die in 315 rounds for DT. This is because the DT allows all the nodes to communicate directly with the BS that causes the nodes to lose energy very quickly, thereby causing the nodes to die. The LEACH protocol shows improvement than the DT, in which the first dead node is found at the round 270 and all the nodes become dead in 714 rounds because of the random selection of $\mathrm{CHs}$. Then metaheuristic search algorithms such as PSO, HSA, and SSA algorithms cause the first node to die at the round 1201, 876, and 424 respectively; and thereby cause all the nodes to die in 1229,906 , and 1637 rounds respectively. The proposed HSHSA CHS, combining the advantages of both HSA and SSA cause all the nodes to die in 1752 rounds. The numerical outcomes are provided in the Table II.

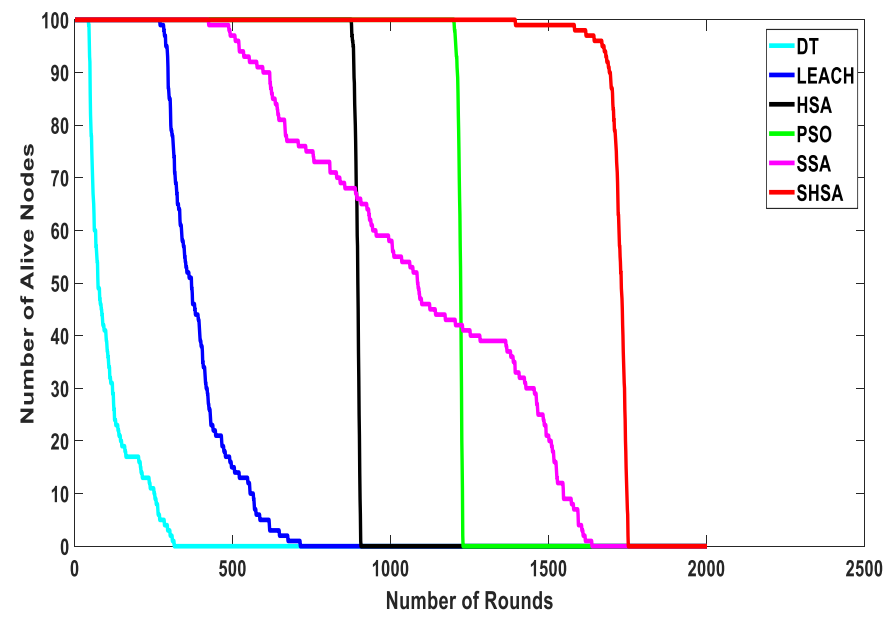

Fig. 4. Comparison of Alive Nodes Obtained for Various Algorithms.

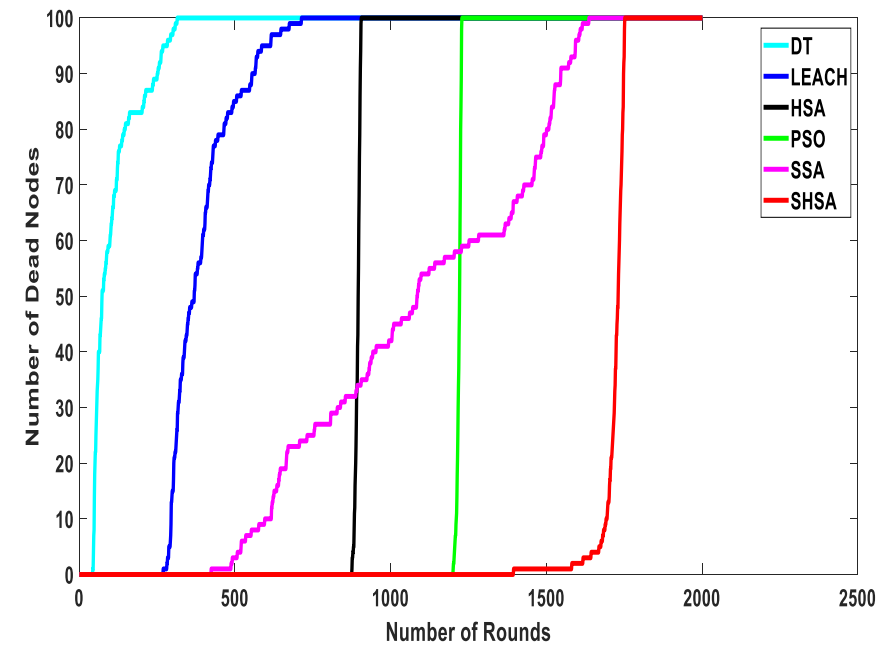

Fig. 5. Comparison of Dead Nodes Obtained for Various Algorithms.

TABLE. II. TABLE OF COMPARISON OF VARIOUS CLUSTER HEAD SELECTION ALGORITHMS

\begin{tabular}{|l|l|l|l|l|}
\hline Algorithm & $\begin{array}{l}\text { First } \\
\text { node } \\
\text { dead }\end{array}$ & $\begin{array}{l}\text { Last } \\
\text { node } \\
\text { dead }\end{array}$ & $\begin{array}{l}\text { Residual energy } \\
\text { (J) after 300 } \\
\text { rounds }\end{array}$ & $\begin{array}{l}\text { Throughput } \\
\text { (bits/round) }\end{array}$ \\
\hline DT & 44 & 315 & 0 & 0 \\
\hline LEACH & 270 & 714 & 9.5661 & 305000 \\
\hline PSO & 1201 & 1229 & 37.6613 & 409600 \\
\hline HAS & 876 & 906 & 33.1616 & 349700 \\
\hline SSA & 424 & 1637 & 33.1022 & 405500 \\
\hline $\begin{array}{l}\text { Proposed } \\
\text { HSHSA }\end{array}$ & 1395 & 1752 & 40.6105 & 415900 \\
\hline
\end{tabular}


Fig. 6 and 10 show the mean residual energy for various sink node positions, and varying count of nodes, respectively. Fig. 7 and 11 show the mean throughput for various sink node positions, and varying count of nodes, respectively. From the bar charts displayed above, the suggested HSHSA outperforms the existing optimization techniques for various positions of the sink node. This is also validated from the standard deviation (SD) of the outcomes obtained. In Fig. 8 and 12, the SD of residual energy is shown for varying sink node positions, and varying count of the nodes respectively. Fig. 9 and 13 show the standard deviation of the throughput for various sink node positions, and varying count of nodes respectively. The standard deviation of residual energy and throughput obtained for the proposed HSHSA are less compared to HSA, SSA, and PSO. This is because, the results of the HSHSA show less deviations, whereas the results of HSA, SSA, and PSO exhibit high deviations. It is inferred from the simulation outcomes that the suggested HSHSA approach of CHS reveals superior performance among other existing cluster head selection techniques.

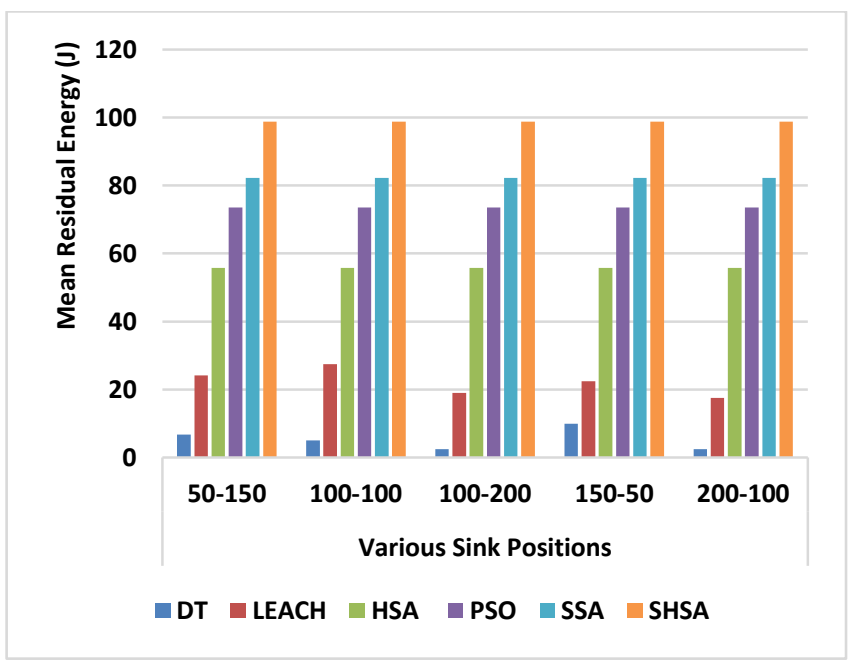

Fig. 6. Mean Residual Energy for Various Positions of the Sink Node.

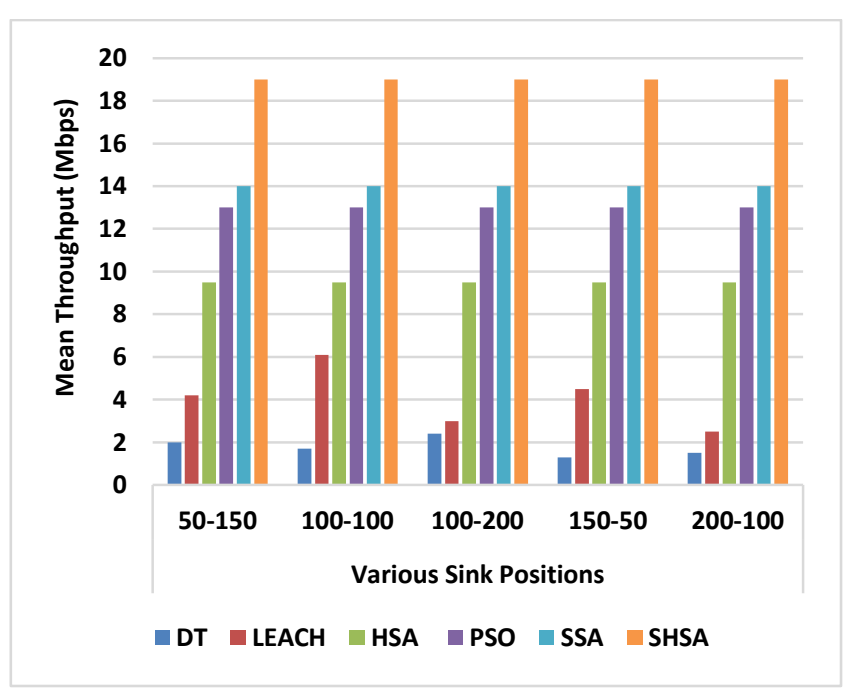

Fig. 7. Mean throughput for Various Positions of the Sink Node.

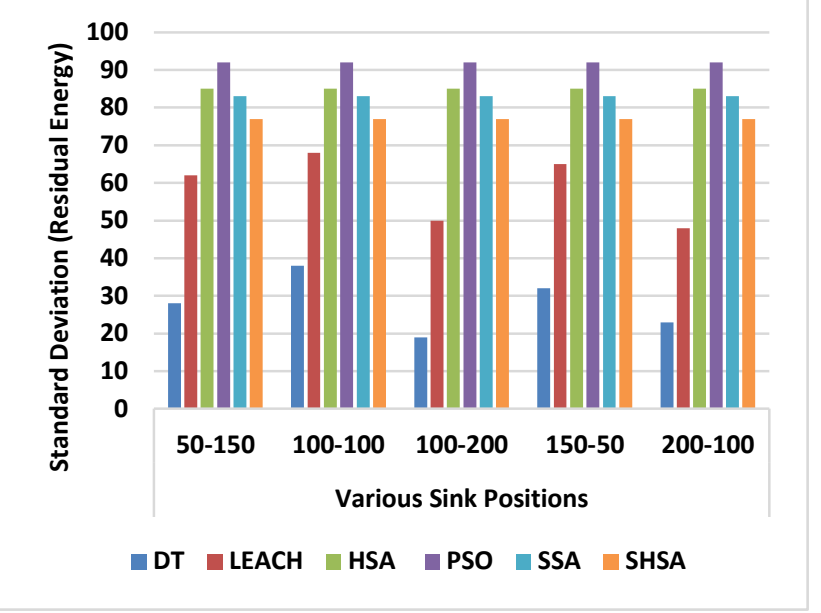

Fig. 8. Standard Deviation of Residual Energy for different Sink Node Positions.

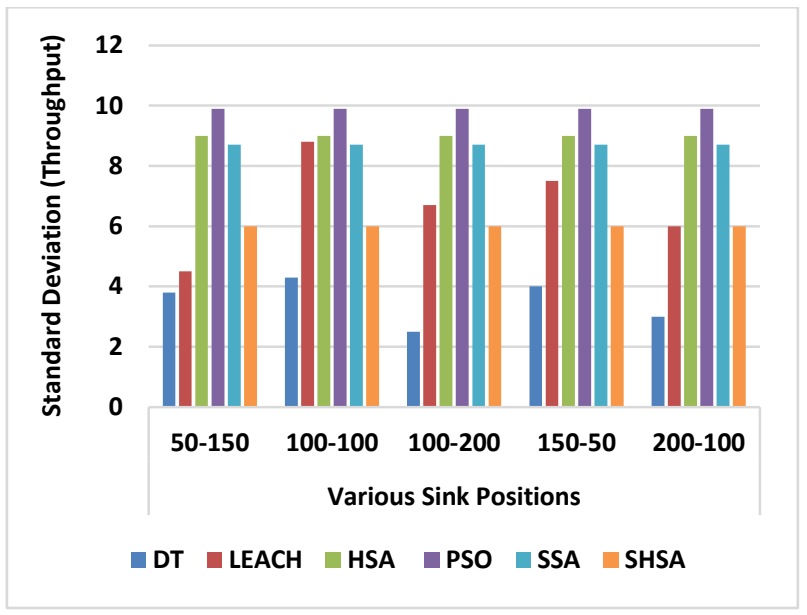

Fig. 9. Standard Deviation of throughput for different Sink Node Positions.

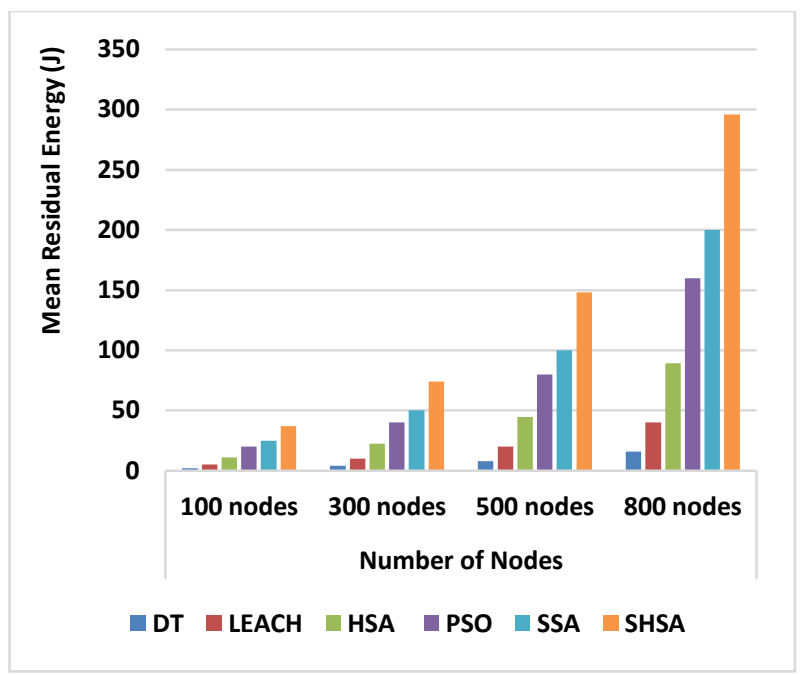

Fig. 10. Mean Residual Energy for Varying Count of Nodes. 


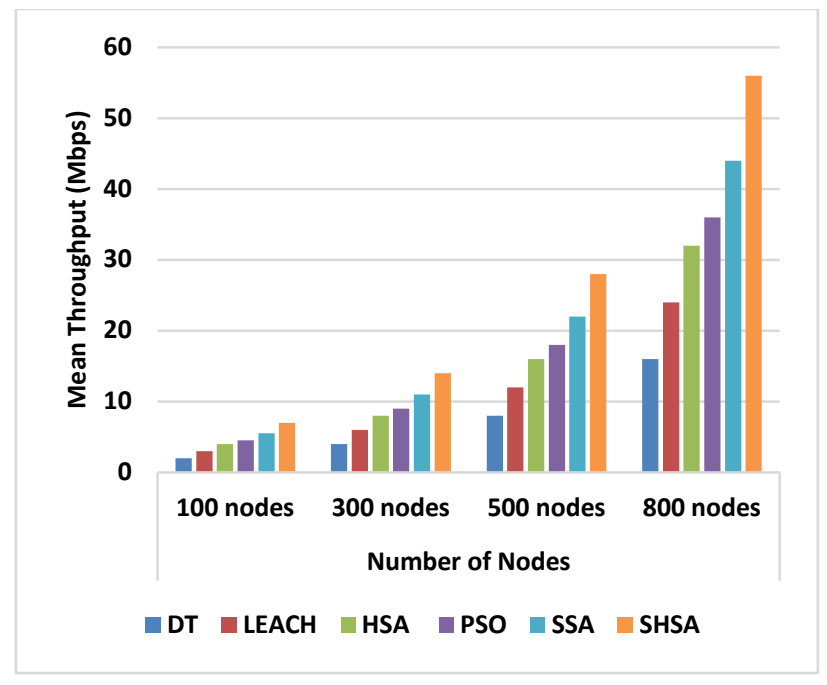

Fig. 11. Mean Throughput for Varying Count of Nodes.

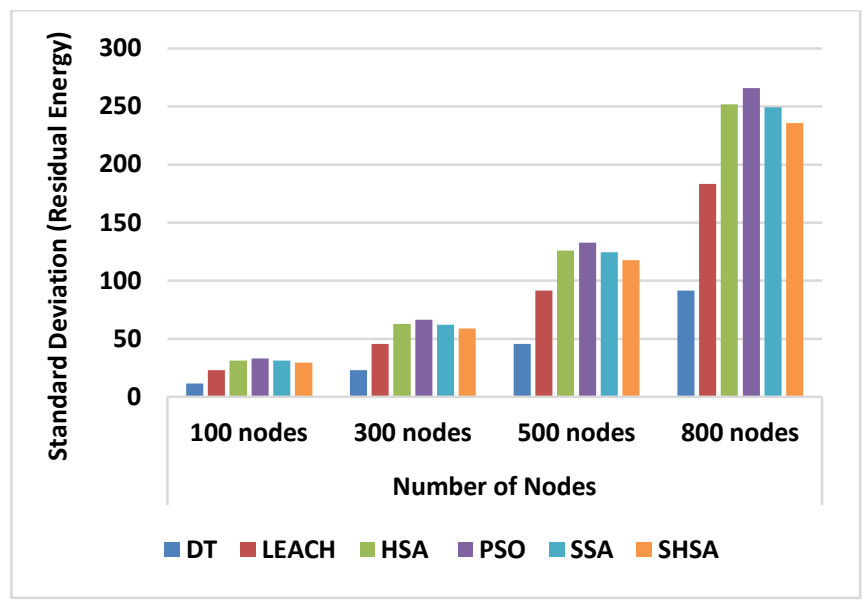

Fig. 12. Standard Deviation of Residual Energy for Varying Count of Nodes.

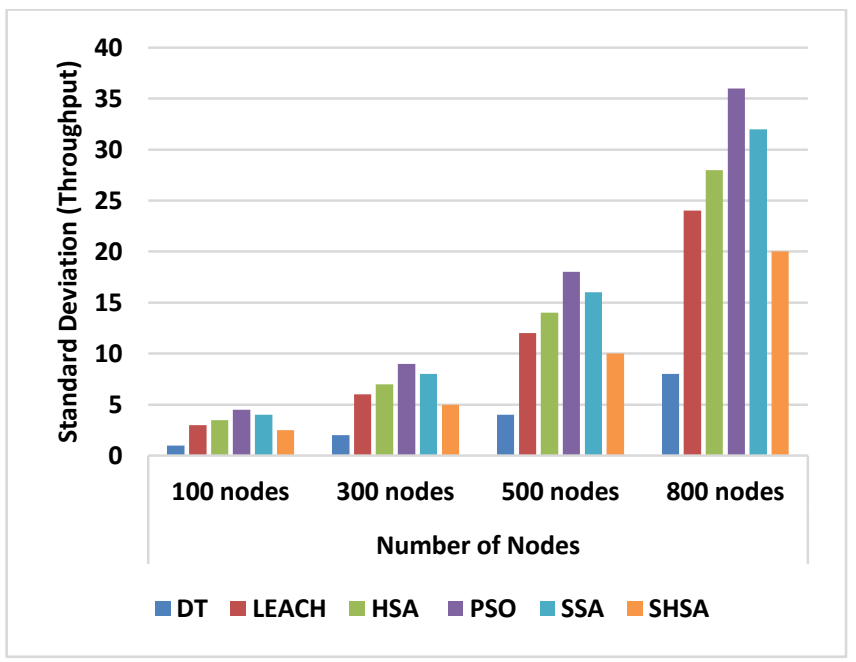

Fig. 13. Standard Deviation of throughput for Varying Count of Nodes

\section{CONCLUSIONS}

This research proposes HSHSA for optimal selection of cluster heads in WSN based on two parameters namely, energy and distance. The natural behavior of the SSA is developed by employing the mechanism of updating locations of the squirrels with predator presence probability. The balance between the exploitation and the exploration is attained by using the gliding constant. The seasonal monitoring prevents the solution of the proposed technique from being bound to the solutions of local optima. The proposed HSHSA is developed by integrating SSA with a high search efficient optimization algorithm called HSA. These advantages make the proposed optimization algorithm offers better performance than the PSO in terms of residual energy and throughput, with an improvement of $85.69 \%$ and $31.02 \%$, respectively. The simulation results prove that the proposed HSHSA is more energy efficient than the existing cluster head selection schemes such as Direct Transmission, LEACH, PSO, HSA, and SSA. The scope for future work lies in the utilization of the different constraints namely, delay and sensing capabilities in different applications.

\section{REFERENCES}

[1] I. F. Akyildiz, Y. Sankarasubramaniam, and E. Cayirci, "A survey on sensor networks", IEEE Communications magazine, 2002, 40(8), pp. 102-114.

[2] Z. Jiang, and A. Wei. "An energy balanced algorithm of LEACH protocol in WSN". International Journal of Computer Science Issues (IJCSI), 2013, 10(1), pp. 354.

[3] P. Azad, and V. Sharma, "Cluster head selection in wireless sensor networks under fuzzy environment". ISRN Sensor Networks ,2013.

[4] A. Abbasi, and M. Younis, "A survey on clustering algorithms for wireless sensor networks". Computer communications, 2007, 30(14-15), pp. 2826-2841.

[5] T. Shankar, S. Shanmugavel, and A. Rajesh, "Hybrid HSA and PSO algorithm for energy efficient cluster head selection in wireless sensor networks". Swarm and Evolutionary Computation, 2016, 30, pp. 1-10.

[6] N. Lavanya, and T. Shankar, "Energy Optimization in Wireless Sensor Network using NSGA-II", 2017.

[7] A. Sampath, and S.Thampi, "An ACO algorithm for effective cluster head selection". arXiv preprint arXiv:1111.6218, 2011.

[8] Anindita Ray and Debashis De, "Energy efficient cluster head selection in wireless sensor network", International Conference on Recent Advances in Information Technology (RAIT), 2012.

[9] M. Nasim, S. Qaisar, and S. Lee, "An energy efficient cooperative hierarchical MIMO clustering scheme for wireless sensor networks". Sensors, 12(1), pp.92-114, 2012.

[10] R. N. Enam, M. Imam, and R. I. Qureshi, "Energy consumption in random cluster head selection phase of WSN". International Proceedings of Computer Science \& Information Tech, 30, pp.38-44, 2012.

[11] S. Nikolidakis, D. Kandris, D. Vergados, and C. Douligeris, "Energy efficient routing in wireless sensor networks through balanced clustering". Algorithms, 6(1), pp.29-42, 2013.

[12] D. C. Hoang, P. Yadav, R. Kumar, and S. K Panda, "Real-time implementation of a harmony search algorithm-based clustering protocol for energy-efficient wireless sensor networks". IEEE transactions on industrial informatics , 2013, 10(1), pp.774-783.

[13] M. Baskaran, and C. Sadagopan, "Synchronous firefly algorithm for cluster head selection in WSN". The Scientific World Journal,2015. 
[14] J. Rejina Parvin, and C. Vasanthanayaki, "Particle swarm optimizationbased clustering by preventing residual nodes in wireless sensor networks". IEEE sensors journal , 2015, 15(8), pp.4264-4274.

[15] R. Sharma, N. Mishra, and S. Srivastava, "A proposed energy efficient distance based cluster head (DBCH) Algorithm: An Improvement over LEACH". Procedia Computer Science, 2015 , 57, pp.807-814.

[16] N. Lavanya, T. Shankar, "A Review on Energy-Efficient Scheduling Mechanisms in Wireless Sensor Networks". Indian Journal of Science and Technology , 2016, (9),pp. 32.

[17] D. Bhatti, N. Saeed, and H. Nam, "Fuzzy c-means clustering and energy efficient cluster head selection for cooperative sensor network". Sensors, 16(9), 2016, pp.1459.

[18] P. S. Rao, P. K. Jana, and H. Banka, "A particle swarm optimization based energy efficient cluster head selection algorithm for wireless sensor networks". Wireless networks , 2017 , 23(7), pp.2005-2020.

[19] P. S. Mann, and S. Singh, “Energy efficient clustering protocol based on improved metaheuristic in wireless sensor networks". Journal of Network and Computer Applications, 2017, 83, pp.40-52.

[20] A. Genta, D. K. Lobiyal, and J.H. Abawajy, "Energy Efficient Multipath Routing Algorithm for Wireless Multimedia Sensor Network". Sensors, 2019, 19(17), pp.3642.

[21] L. H. Correia, T. Heimfarth, G.M. Pereira, V. F. Silva, and J. L Santana, "Radio channel model of wireless sensor networks operating in $2.4 \mathrm{GHz}$ ISM band". INFOCOMP, 2010, 9(1), pp.98-106.

[22] Z. W. Geem, J. H Kim, G. V. Loganathan, "A new heuristic optimization algorithm: harmony search”. Simulation ,2001, 76(2), pp.60-68.

[23] M. Jain, V. Singh, and A. Rani, “ A novel nature-inspired algorithm for optimization: Squirrel search algorithm". Swarm and evolutionary computation , 2019, 44, pp.148-175.
[24] J. W. Bahlman, S. M. Swartz, D. K. Riskin, and K.S. Breuer, "Glide performance and aerodynamics of non-equilibrium glides in northern flying squirrels (Glaucomys sabrinus)". Journal of The Royal Society Interface, 10(80), 2010, pp.794.

[25] U. M. Norberg, "Evolution of vertebrate flight: an aerodynamic model for the transition from gliding to active flight". The American Naturalist, 1985, 126(3),pp. 303-327.

[26] W. B. Heinzelman, A. P. Chandrakasan, and H. Balakrishnan, “An application-specific protocol architecture for wireless microsensor networks". IEEE Transactions on wireless communications, 2002, 1(4), pp. 660-670.

[27] J. Tillett, R. Rao, and F. Sahin, "Cluster-head identification in ad hoc sensor networks using particle swarm optimization". In IEEE International Conference on Personal Wireless Communications, 2002, pp. 201-205.

[28] Z. W. Geem, J. H. Kim, and G.V Loganathan, "A new heuristic optimization algorithm: harmony search. Simulation",2001, 76(2), pp.60-68.

[29] M. Jain, V. Singh, and A. Rani, "A novel nature-inspired algorithm for optimization: Squirrel search algorithm". Swarm and evolutionary computation ,44, 2019, pp.148-175.

[30] T. Senthil Murugan and Amit Sarkar," Optimal cluster head selection by hybridisation of firefly and grey wolf optimisation", Int. J. Wireless and Mobile Computing, Vol. 14, No. 3, 2018

[31] Z.Han, J. Wu, J. Zhang, L. Liu, and K Tian, 'A general self-organized tree-based energy-balance routing protocol for wireless sensor network', IEEE Transactions on Nuclear Science, Vol. 61, No. 2, pp.732-740, 2014.

[32] A. Puggelli, et al. 'Routing-aware design of indoor wireless sensor networks using an interactive tool', IEEE Systems Journal, Vol. 9, No. 3, pp.714-727, 2015. 